\title{
The Effects of Exercise and Rest Breaks on Musculoskeletal Discomfort during Computer Tasks: An Evidence-Based Perspective
}

\author{
Ronald De Vera BARRedo ${ }^{1)}$, Kelly MAHON ${ }^{2)}$ \\ 1) The College of Health Sciences, Tennessee State University: 3500 Jhon A Merritt Bonlevard, \\ Nashville TN 37209, USA. \\ ${ }^{2}$ The Graduate program in Physical Therapy, Arkansas State University
}

\begin{abstract}
OBJECTIVE: To review the strength of research evidence on the effects of exercise and rest breaks on musculoskeletal discomfort during computer tasks and compare the evidence with clinical guidelines. SIGNIFICANCE: The review of research evidence and its comparison with current clinical guidelines provide clinicians with knowledge to make clinically sound decisions in the care and management of individuals with musculoskeletal discomfort during computer tasks. METHODS: Articles from Pubmed, Ovid and references of relevant articles were reviewed for research design and internal validity. Grades of evidence were assigned based on the aggregate strength of articles for each intervention. RESULTS: Fifteen articles (one on exercise, seven on rest breaks, five examining both) met the inclusion criteria. Exercise and rest breaks were each assigned a grade of C. CONCLUSIONS: Evidence supports the use of exercise and rest breaks in reducing musculoskeletal discomfort in computer tasks. The research evidence suggests no additional benefits of exercise over rest breaks alone. Research evidence concurs with the clinical guidelines recommended by OSHA and the Official Disability Guidelines.
\end{abstract}

Key words: Computers, Musculoskeletal, Exercise

(This article was submitted Nov. 13, 2006, and was accepted Jan. 18, 2007)

\section{INTRODUCTION}

Computer use frequently causes musculoskeletal discomfort ${ }^{1-13)}$ which, according to a survey of the literature, may include muscle tension, muscle fatigue, paresthesia, pain, and/or physical strain in soft tissues and bones ${ }^{7,14-17)}$. The incidence of musculoskeletal discomfort in computer users is as high as $50 \%{ }^{5,18)}$. Musculoskeletal discomfort has been reported by computer users, either separately or in aggregate, in the neck, shoulders, elbows, wrists, hands, back, legs, buttocks, ankles, feet, and

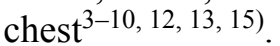

Many interventions have been implemented to decrease musculoskeletal discomfort in computer tasks. The interventions include individual, organizational, and ergonomic modifications. Individual interventions include routine exercise $^{16,19,20)}$ and smoking cessation ${ }^{19,21)}$. The organizational modifications include: increased work task variety, decreased hours of computer use per day ${ }^{3,4,21,22)}$, increased rest break opportunities,

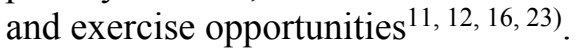

Ergonomic changes to the computer workstation are the most frequently employed intervention. Ergonomic interventions are not sufficient for completely eliminating musculoskeletal discomfort ${ }^{7)}$. In some cases, ergonomic 
interventions have caused no changes in musculoskeletal discomfort. Therefore, work organizational factors must be incorporated.

Workstation exercises and supplemental rest breaks are examples of organizational interventions. They are inexpensive and advantageous interventions aimed at decreasing musculoskeletal discomfort in computer tasks ${ }^{10)}$. As separate interventions, exercises and rest breaks have been attributed to increased productivity in computer tasks ${ }^{16)}$ such as work with computers, video display terminals (VDT) and data entry tasks.

Many exercises have been recommended to decrease musculoskeletal discomfort, with varying degrees of efficacy ${ }^{24,25)}$. They consist primarily of stretching and relaxation techniques, which can be performed at the computer workstation (with the exception of walking) in ten minutes or less.

Rest breaks have also been recommended to decrease musculoskeletal discomfort. Toward this end, studies have reported that limited opportunities for rest breaks are a major causative factor in many musculoskeletal disorders in computer users ${ }^{9,10)}$. Rest breaks, rest, and breaks in this appraisal are defined as cessation of computer tasks. During the rest breaks participants are not required to remain motionless; however, they must not perform a specific exercise protocol.

This study examines the effects of exercise (irrespective of technique) and rest breaks (irrespective of length and frequency) on musculoskeletal discomfort during computer tasks. For the purposes of this project, musculoskeletal discomfort is defined as a constellation of signs and symptoms that include muscle tension, paresthesia, pain and/or physical strain in soft tissues and bones. More specifically, this study reviews the strength of the current research evidence on exercise and rest breaks and compares each intervention's strength with current clinical guidelines, to provide clinicians with ample evidence and working knowledge to make clinically sound decisions in the care and management of individuals with musculoskeletal discomfort during computer tasks.

\section{METHODS}

\section{Search strategy}

Three search strategies were used in a literature search. The first search strategy was conducted using Pubmed with the following limits: publication date 1990-April 2005, clinical query, therapy, broad-sensitive search and English. The following search terms were used in the literature search of Pubmed: "Computer or VDT and musculoskeletal and exercise," "Computer or VDT and musculoskeletal and rest," and "Computer or VDT and musculoskeletal and breaks."

The second search strategy was conducted using the Ovid portal with the following limits: 1990April 2005, therapeutics, English, and human. The following databases were searched through the Ovid portal: Cumulative Index to Nursing and Allied Health Literature (CINAHL 1990-week 2 April 2005), Ovid Medline (1996-week 1 April 2005), Ovid Medline in Process and Non-Indexed Citations (1996-April 1, 2005), and all Evidencebased Medicine Reviews (Database of Abstracts of Reviews of Effects, Cochrane Database of Systematic Reviews, ACP Journal Club, Cochrane Central Register of Controlled Trials).

The third search strategy reviewed the references of relevant articles obtained through the first and second search strategies. Conference proceedings and articles published prior to 1990 were also included if they were referenced in two or more relevant articles.

This literature appraisal was restricted to articles which analyze rest breaks or exercise. Exercise and rest breaks had to be analyzed independent of other variables to be included. Articles that examined multiple occupations were excluded if all occupations did not involve computer use. Articles had to have clearly defined methods to be included in this study. The articles had to describe the parameters of rest breaks. Studies with exercise protocols lasting longer than 10 minutes were excluded. Exercise breaks are often considered breaks supplemental to the breaks required by law. Employers are unlikely to permit employees breaks supplemental to perform exercises if the protocols require longer than ten minutes to complete.

Review articles other than meta-analysis or systematic reviews were excluded because they are primarily literature reviews. Studies with participants from multiple occupations, in which not all participants used computers were excluded.

\section{Evaluation of research evidence}

Each article was reviewed and the strength of evidence was evaluated using the American Academy for Cerebral Palsy and Developmental 
Table 1. Research designs ${ }^{26)}$

\begin{tabular}{|c|c|c|c|}
\hline Level & Non-empirical & Group Research & Outcomes Research \\
\hline I & & $\begin{array}{l}\text { - Systematic review of } \\
\text { randomized } \\
\text { controlled trials } \\
\text { (RCT's) } \\
\text { - Large RCT (with } \\
\text { narrow confidence } \\
\text { intervals) }(\mathrm{N}>100)\end{array}$ & \\
\hline II & & $\begin{array}{l}\text { - Systematic review of } \\
\text { cohort studies } \\
\text { - Smaller RCT's (with } \\
\text { wider confidence } \\
\text { intervals) }(\mathrm{N}<100)\end{array}$ & $\begin{array}{l}\text { - Outcomes research (very } \\
\text { large ecologic studies) }\end{array}$ \\
\hline III & & $\begin{array}{l}\text { - Systematic review of } \\
\text { case-control studies } \\
\text { - Cohort study with } \\
\text { concurrent control } \\
\text { group }\end{array}$ & \\
\hline IV & & $\begin{array}{l}\text { - Cohort study without } \\
\text { concurrent control } \\
\text { group (e.g. with } \\
\text { historical control } \\
\text { group) } \\
\text { - Case series } \\
\text { - Case-control study }\end{array}$ & \\
\hline V & $\begin{array}{l}\text { - Expert opinion } \\
\text { - Expert opinion } \\
\text { based on theory or } \\
\text { physiologic research } \\
\text { - Case study or report } \\
\text { - Bench research } \\
\text { - Common } \\
\text { sense/anecdotes }\end{array}$ & & \\
\hline
\end{tabular}

Medicine (AACPDM) method ${ }^{26)}$. Developed by the AACPDM Treatment Outcomes Committee, this method is a modification of the Centre for Evidence-Based Medicine (CEBM) method $^{27,28)}$ created by Dave Sackett and colleagues. The level of evidence for each article was determined by its research design (Table 1) and internal validity (Tables 2 and 3$)^{26)}$.

The AACPDM method has been used in cerebral palsy $^{26,29)}$ and knee osteoarthritis ${ }^{30)}$. This method has been determined as an appropriate method for examining evidence of the effects of exercise and rest breaks in computer tasks by Johanna Darrah of the AACPDM Treatment Outcomes Committee (J. Darrah, PhD, PT, [johanna.darrah@ualberta.ca], email, Wednesday, April 27, 2005).

After evaluating the strength of each article using the AACPDM method, the aggregate strength of each intervention was evaluated using the criteria outlined by Sackett et al. ${ }^{28)}$ (Table 4) and grades of recommendation ${ }^{27,28)}$ (Table 5) were given and subsequently compared with existing clinical guidelines.

There were two reviews of each article. The first review was conducted by the primary author. The second reviewer reviewed the appraisals conducted by the first reviewer. In the event that the two reviewers did not agree, the reviewers meet to discuss their findings to gain consensus. This method increased the validity of the review process. 
Table 2. Internal validity questions ${ }^{25)}$

1. Were inclusion and exclusion criteria of the study population well described and followed?

2. Was the intervention well described and was there adherence to the intervention assignment? (For 2-group designs, was the control exposure also well described?)

3. Were the measures used clearly described? Were they valid and reliable for measuring the outcomes of interest?

4. Was the outcome assessor unaware of the intervention status of the participants (i.e., blind assessment)?

5. Did the authors conduct and report appropriate statistical evaluation including power calculations?

6. Were dropout/loss to follow-up reported and less than 20\%? For 2-group designs, was dropout balanced?

7. Considering the study design, were appropriate methods for controlling confounding variables and limiting potential biases used?

Table 3. Internal validity score ${ }^{25)}$

\begin{tabular}{cll}
\hline S & Strong (well conducted) & 6 to 7 \\
M & Moderate (fairly conducted) & 4 to 5 \\
W & Weak (poorly conducted) & 3 or less \\
\hline
\end{tabular}

\section{RESULTS}

The literature search revealed three articles on the effects of exercise breaks on musculoskeletal discomfort during computer tasks. Seven published articles on the effects of rest breaks on musculoskeletal discomfort during computer tasks were obtained through the literature search. Five articles that examined the effects of both exercise and rest breaks on musculoskeletal discomfort during computer tasks were obtained from the literature search. Reviews of each article, including justification for the assigned level of evidence are given below.

\section{Articles on exercise}

Fenety and Walker ${ }^{11)}$ examined the short term effects of a revised Dataspan exercise program on eleven directory assistance operators. During the experimental period of three to five day shifts, participants took one workstation exercise break every 30 minutes. Musculoskeletal discomfort, as measured by the Body Part Discomfort Scale, increased over time during the pre-exercise and exercise testing sessions. However, discomfort was significantly higher during testing periods when participants were not exercising. In-chair movement was significantly greater in the exercise test session than in the pre-exercise test session, indicating decreased postural immobility in the exercise condition.

The research design employed by Fenety and Walker ${ }^{11)}$ was a non-randomized clinical trial in which participants served as their own controls. According to Bob Phillips of the Centre for Evidence-Based Medicine, non-randomized clinical trials are best understood as cohort studies (Bob Phillips, MD [bob.phillips@doctors.org.uk], email, Tuesday, September 6, 2005). This design qualifies as level of evidence IV. This study had moderate internal validity. The lack of reliability data for the Body Part Discomfort Scale lowered the internal validity ${ }^{26)}$.

Saltzman ${ }^{31)}$ examined the impact of Stretch Break, an ergonomic software program, via a computer assisted survey. Each stretching session consisted of several stretches lasting 1-2 minutes. The participants reported that short stretch breaks were effective in reducing stiffness, muscle ache, and stress. They credited increased awareness of the need for frequent mini-breaks and proper workstation ergonomics to the Stretch Break program. Twenty-three percent of participants reported increased productivity and enjoyment working at their personal computer (PC) when using Stretch Break.

The level of evidence based on the research design of this study was $\mathrm{V}$ because the study employed a survey for all data collection. The internal validity was weak in this study because the measures used have not been proven to be reliable, appropriate statistical evaluations were not 
Table 4. Grades of recommendation and levels of evidence ${ }^{26)}$

\begin{tabular}{ccl}
\hline Grade & $\begin{array}{r}\text { Level of } \\
\text { Evidence }\end{array}$ & Therapy/Prevention, Aetiology/Harm \\
\hline A & 1a & SR (with homogeneity) of RCTs \\
& 1b & Individual RCT (with narrow Confidence Interval) \\
& 1c & All or none \\
\hline B & 2a & SR (with homogeneity) of cohort studies \\
& 2b & Individual cohort study (including low quality RCT; e.g., <80\% follow-up) \\
& 2c & "Outcomes" Research \\
\hline C & $3 \mathrm{a}$ & SR (with homogeneity) of case-control studies \\
& $3 \mathrm{~b}$ & Individual Case-Control Study \\
\hline D & 4 & Case-series (and poor quality cohort and case-control studies) \\
\hline E & 5 & Expert opinion without explicit critical appraisal, or based on physiology, \\
& & bench research or "first principles" \\
\hline
\end{tabular}

Table 5. Grades of recommendation ${ }^{26)}$

$\begin{array}{ll}\text { A } & \text { Consistent level } 1 \text { studies } \\ \text { B } & \text { Consistent level } 2 \text { or } 3 \text { studies or extrapolations from level } 1 \text { studies } \\ \text { C } & \text { Level } 4 \text { studies or extrapolations from level } 2 \text { or } 3 \text { studies } \\ \text { D } & \text { Level } 5 \text { evidence or troublingly inconsistent or inconclusive studies of any level }\end{array}$

conducted, $43 \%$ of participants dropped out of the study, and confounding variables and potential biases were not limited ${ }^{26,31)}$.

Thompson ${ }^{14)}$ conducted a study evaluating the effects of exercise on musculoskeletal discomfort and productivity of 85 data entry operators. During eight-hour workdays, employees were required to perform 6.5 hours of "logged on" time. Two 5minute exercise breaks were added to the traditional work day. Employees were encouraged to take the 5-minute exercise breaks in mid-morning and midafternoon, exercise during their normal rest breaks instead of remaining passive, perform the exercises on personal time at work or home, emphasize exercises which seemed to cause the most relaxation and discomfort relief, and take additional mini-breaks of 20-30 seconds to stretch, flex, and shake muscles if discomfort developed. The operators reported reduced discomfort at work and a generally improved physical condition outside of work. There were no new workman's compensation claims one year after introduction of the exercise program. Productivity increased 25\% over the first four months of the exercise program.

The research design level of evidence was $\mathrm{V}$ in Thompson's ${ }^{14)}$ study because formal statistical analyses were not performed. Musculoskeletal discomfort was not formally assessed before or during the exercise program. The internal validity was weak in this study because the measures used were not clearly described, dropout was not reported, and confounding variables were not controlled by permitting large variations in exercise protocol amongst participants ${ }^{26)}$.

Table 6 provides a summary of the analysis on research design and internal validity of each article on exercise using the AACPDM tool ${ }^{26)}$. A grade of recommendation, based on the CEBM method ${ }^{27,28)}$, is provided for exercise as an intervention for musculoskeletal discomfort during computer tasks.

\section{Articles on rest breaks}

Boucsein and Thum ${ }^{32)}$ performed a field study to investigate physical comfort and emotional wellbeing during two different work-rest schedules for complex computer-mediated work. Each participant worked under a long break schedule of 15 minutes break after 100 minutes of work on one day and under a short break schedule of 7.5 minutes break after 50 minutes of work on another day. The total break time each day was 82.5 minutes, which included the lunch break. Musculoskeletal 
Table 6. Level of evidence summary-Exercise

\begin{tabular}{|c|c|c|c|c|c|c|c|c|c|c|c|}
\hline \multirow[b]{2}{*}{ Study } & \multicolumn{7}{|c|}{ Internal validity questions } & \multirow{2}{*}{$\begin{array}{l}\text { Internal } \\
\text { validity } \\
\text { sum }\end{array}$} & \multirow{2}{*}{$\begin{array}{l}\text { Internal } \\
\text { validity } \\
\text { score* }\end{array}$} & \multirow{2}{*}{$\begin{array}{c}\text { Research } \\
\text { design }\end{array}$} & \multirow{2}{*}{$\begin{array}{l}\text { Level of } \\
\text { evidence }\end{array}$} \\
\hline & 1 & 2 & 3 & 4 & 5 & 6 & 7 & & & & \\
\hline \multicolumn{12}{|l|}{ Articles concluding exercise is beneficial } \\
\hline Balci, 2003 ${ }^{10)}$ & Yes & Yes & Yes & No & Yes & Yes & Yes & 6 & $\mathrm{~S}$ & II & II-S \\
\hline Fenety, 2002 ${ }^{11)}$ & Yes & Yes & No & No & Yes & Yes & Yes & 5 & M & IV & IV-M \\
\hline Henning et al., 1997 small worksite ${ }^{12)}$ & Yes & Yes & No & No & Yes & No & No & 3 & $\mathrm{~W}$ & II & II-W \\
\hline Saltzman, $1998^{32)}$ & Yes & Yes & No & No & No & No & No & 2 & W & $\mathrm{V}$ & V-W \\
\hline Sundelin \& Hagberg, 198940) & Yes & Yes & Yes & No & No & Yes & Yes & 5 & M & IV & IV-M \\
\hline Swanson \& Sauter, 199241) & Yes & Ye & No & No & No & No & Yes & 3 & $\mathrm{~W}$ & II & II-W \\
\hline Thompson, 1990 & Yes & Yes & No & No & No & No & No & 2 & $\mathrm{~W}$ & $\mathrm{~V}$ & V-W \\
\hline van den Heuval et al., 2003 & Yes & Yes & No & No & Yes & Yes & Yes & 5 & M & I & $\mathrm{I}-\mathrm{M}$ \\
\hline \multicolumn{12}{|c|}{ Article concluding exercise is not beneficial } \\
\hline Henning et al., 1997 large worksite ${ }^{12)}$ & Yes & Yes & No & No & No & No & No & 2 & W & IV & IV-W \\
\hline
\end{tabular}

discomfort and exhaustion increased significantly under both break schedules. The degree of discomfort was not significantly different between the two work-rest schedules. Emotional well-being was significantly better under short break schedule in the morning and significantly better under the long break schedule in the afternoon.

A crossover study design without randomization was used by Boucsein and Thum ${ }^{32}$. No concurrent control group was used in this study. Studies using non-randomized crossover designs are best understood as small cohort studies (Bob Phillips, MD, [bob.phillips@doctors.org.uk], email, Tuesday, September 6, 2005). This study employed a level IV research design. This study had weak internal validity. The measures used have not been proven reliable, power calculations were not performed, confounding variables were not controlled, and loss to follow up was not reported $^{26)}$.

Ferreira et al. ${ }^{33)}$ performed a retrospective study on the incidence of musculoskeletal disorders of the wrists and hands in an at-risk population of female customer service operators. The variables studied included: age, seniority, mean daily regular work time, overtime per operator, time pressure at work, work-rest schedule, management status, personnel training on posture and muscle stretching, and ergonomic hazards. The variables that significantly correlated with upper extremity musculoskeletal disorders were time pressure at work and work-rest schedule. The effects of exercise examined by Ferreira et al. were not analyzed independent of other variables. Therefore, the exercise component of their study was not included in this evidencebased review.

A case-control study design, level of evidence IV, was employed in the study by Ferreira et al..$^{33)}$ The internal validity of the study had moderate strength. Confounding variables were not controlled and dropout was greater than twenty percent ${ }^{26)}$.

Galinsky et al. ${ }^{9)}$ compared the effects of a conventional and supplemental work-rest schedule on musculoskeletal discomfort, eyestrain, and performance in data entry workers. The conventional schedule consisted of a 15-minute break in the middle of the first and second halves of the shift, in addition to a 30-minute lunch break. The supplemental schedule included the same breaks as the conventional schedule, in addition to a 5-minute break during every hour which did not include a break in the conventional schedule. Musculoskeletal discomfort increased during the work periods for both schedules. However, the degree of discomfort was lower in the supplemental schedule in the eyes, neck, back, buttocks, entire right upper extremity, and the left shoulder, upper arm, and elbow. There was no significant difference in productivity between the two schedules. 
A randomized crossover design with less than 100 participants was used in Galinsky's study. According to the $\mathrm{CEBM}^{34)}$, this was a level of evidence II research design. There was moderate internal validity in this study because the tool for measuring musculoskeletal discomfort has not been proven reliable and $58 \%$ of participants dropped out of the study ${ }^{9,26)}$.

Henning et al., 1994 ${ }^{35)}$, compared mood state, performance, and discomfort in a regimented rest break system and a compensatory rest break system. In the regimented break system, participants received a 20 -second computer administered break after every 5 minutes of work. In the compensatory rest break system, spontaneous pauses lasting 3 seconds or longer were deducted from the length of the computer administered rest breaks. Participants in the compensatory rest break condition received a 20 -second computer administered break after every 5 minutes of work only if their spontaneous rest pauses totaled less than 17 seconds. Spontaneous pauses lasting longer than 3 seconds occurred, on average, in 4 out of 9 possible 5 -minute work segments. Discomfort increased during the work period under both conditions. However, back discomfort was significantly lower in the compensatory break system. There was no significant difference between the two break systems in the other body regions, mood state, or performance.

A non-randomized clinical trial, level of evidence IV research design (Bob Phillips, MD, [bob.phillips@doctors.org.uk], email, Tuesday, September 6, 2005), was performed in Henning's study. This study had weak internal validity because the measures used for musculoskeletal discomfort have not been proven reliable, dropout was not reported, and potential biases were not limited due to non-random group assignment ${ }^{26,35)}$.

McLean et al. ${ }^{13)}$ evaluated the impact of three different microbreak schedules on musculoskeletal discomfort, EMG activity, and productivity in computer operators. Participants were assigned to one of the following microbreak schedules: 20minute interval group (30-second microbreak every 20 minutes), 40-minute interval group (30-second microbreak every 40 minutes), or the control group (breaks taken whenever needed). Musculoskeletal discomfort increased over time during the recording sessions for all protocols. Decreased musculoskeletal discomfort was reported in all body regions studied under both microbreak protocols compared to the no-break protocol. The 20-minute interval caused the greatest decrease in discomfort. Higher mean frequency (MNF) cycling was present in all microbreak protocols at the wrist extensors, in the control and 40-minute interval groups at the neck, and in the 20- and 40-minute interval groups at the back. No significant changes in MNF cycling were present at the shoulder. Productivity was not significantly affected by the microbreaks.

A randomized controlled clinical trial was performed in this study. RCT's with less than 100 participants, as in the study by McLean et al. ${ }^{13)}$, qualify as level of evidence II research design. This study had strong internal validity ${ }^{26}$.

In the 1986 study by Sundelin et al. ${ }^{36)}$, the impact of spontaneous pauses and micro pauses on musculoskeletal discomfort and muscular load during VDT word-processing tasks was evaluated. Two 3-6 hour work sessions with spontaneous pauses, and one 3-4 hour work session with short pauses of 14 seconds were introduced ten times per hour. Participants took diverting pauses during which they left the workstation and short passive pauses. Increased discomfort was reported after all work sessions. Differences in discomfort between the two rest schedules were present only in the eyes. EMG recordings of the middle and lower trapezius revealed no difference in muscular load between the two rest schedules.

The study of Sundelin et al. employed a nonrandomized crossover research design. The level of evidence, based on research design, was IV in this study (Bob Phillips, MD, [bob.phillips@doctors.org.uk], email, Tuesday, September 6, 2005). The internal validity of this study was limited to moderate because power calculations were not performed ${ }^{26,36)}$.

Zwahlen et al. ${ }^{37)}$ examined the effects of rest breaks and task type on musculoskeletal discomfort and productivity in continuous VDT work. The subjects received a 15-minute rest break in the middle of the morning and afternoon sessions, in addition to a 45-minute lunch break. Musculoskeletal discomfort increased throughout the day. However, the authors concluded rest breaks are highly beneficial for reducing musculoskeletal discomfort. Visual discomfort, which also increased throughout the day, was significantly less than musculoskeletal discomfort. There was minimal difference in discomfort 
between the task types. Productivity was slightly higher after the rest breaks.

The research design level of evidence was $\mathrm{V}$ in this study by Zwahlen et al. ${ }^{37}$ ) because formal statistical analyses were not performed. The internal validity was weak because the measures used have not been proven reliable, appropriate statistical evaluations were not performed, and confounding factors were not sufficiently controlled $^{26)}$.

Table 7 provides a summary of the analysis on research design and internal validity of each article on rest breaks using the AACPDM tool ${ }^{26)}$. A grade of recommendation, based on the CEBM method ${ }^{27,28)}$, is provided for rest breaks as an intervention for musculoskeletal discomfort during computer tasks.

\section{Articles on both rest and exercise}

Balci and Aghazadeh ${ }^{10)}$ compared different work-rest schedules in data entry and simple mental arithmetic tasks. A 60-minute work/10-minute rest, a 30-minute work/5-minute rest, and a 15 -minute work/microbreak were compared. Under the 15minute/ microbreak schedule participants received three 30 -second breaks and one 3-minute break per hour in addition to a 14-minute break after 2 hours. Exercises were performed during all breaks for each work/rest schedule, except the 30 -second breaks. Neck, low back, and chest discomfort were significantly lower in the 15-minute/microbreak schedule than the other schedules. The highest speed, accuracy, and performance was achieved in the 15-minute/microbreak schedule.

Balci and Aghazadeh ${ }^{10)}$ employed a randomized crossover design with less than 100 participants. The level of evidence was II based on research $\operatorname{design}^{34)}$. This study had strong internal validity ${ }^{26)}$.

The effects of short rest breaks and exercise on musculoskeletal discomfort, mood state, and productivity at two field sites was examined in the 1997 study by Henning et al. ${ }^{12)}$. The operators conventional rest-break condition (control) was compared to a rest-break-only condition and a restbreak-plus-exercise condition. The control condition included a 30-minute lunch break, and a 15-minute break in the middle of the morning and afternoon. Operators had the option to split their 15-minute breaks into three 5-minute breaks. The experimental break condition consisted of one 3minute and three 30 -second rest breaks per hour, in addition to the conventional breaks. The exercises required 15 seconds each to complete. Participants in the exercise and rest-break conditions adhered to the same break schedule as in the rest-break-only condition. During the 30 -second breaks participants performed one stretching exercise, and at least two exercises during the 3-minute breaks. At the large work site, the decrease in musculoskeletal discomfort in both treatment groups was not significant. Leg, feet, and eye comfort improved under both treatment conditions at the small work site. The improvement was only significant in the breaks and exercise condition. Mood state did not change at either worksite. There were no significant differences in productivity at the large work site. Productivity increased at the small worksite in both treatment conditions. However, the improvement was only significant in the breaks and exercise condition.

A between-participants randomized controlled clinical trial, level of evidence II, was performed at the large worksite ${ }^{34)}$. A non-randomized crossover research design, level of evidence IV, was used at the small worksite (Bob Phillips, MD, [bob.phillips@doctors.org.uk], email, Tuesday, September 6, 2005). The internal validity was weak at both worksites because the measure used for musculoskeletal discomfort has not been proven reliable, and dropout rate was high ${ }^{12,26)}$.

Sundelin and Hagberg ${ }^{38)}$, in their 1989 study, compared the effects of three different pause types on musculoskeletal discomfort and EMG activity during VDU work. Active pauses, diverting pauses, and passive pauses were each incorporated into separate work periods. Pauses were taken once every six minutes, for 15-20 seconds, during each work period. Discomfort ratings after each work period were very low. There was a tendency, but no statistical significance, toward greater discomfort after the work period involving passive pauses than after the work periods involving active and diverting pauses. Static muscle load did not differ between the three pause types. However, the pattern of muscle activity changed significantly in work periods with active pauses. As a nonrandomized crossover design, this research design qualified as level of evidence IV (Bob Phillips, MD, [bob.phillips@doctors.org.uk], email, Tuesday, September 6, 2005). The internal validity of this study was limited to moderate because power calculations were not performed ${ }^{26,38)}$.

Swanson and Sauter ${ }^{39)}$ examined musculoskeletal 
discomfort, mood state, and productivity of 37 healthy data entry operators. Both groups received the following rest break schedule: 45-minute lunch, 10-minute breaks after 100 minutes of work in the morning and afternoon, 30-second breaks every 10 minutes during the morning and afternoon, 3minute breaks at 50-minute intervals in the morning, and breaks after 30,65, and 150 minutes in the afternoon. During the 3-minute and 30second breaks, the exercise break group performed exercises while the passive break group sat passively. Musculoskeletal discomfort increased in both conditions throughout the workday. The greatest increases in discomfort were reported in the back, neck, and throughout the right upper extremity. There were no differences between groups in musculoskeletal discomfort or mood state. Productivity, which was measured continuously throughout each work session, decreased more significantly during the afternoon in the passive group than in the exercise break group, as revealed by post hoc analyses.

Swanson and Sauter ${ }^{39)}$ conducted a randomized controlled clinical trial. This study presents a level of evidence II research design. The internal validity was weak because the measures used were not clearly described, power calculations were not conducted, and dropout was not reported ${ }^{26)}$.

Van den Heuval et al. ${ }^{15)}$ investigated the effects of software-stimulated rest breaks and exercise on computer workers with neck or upper extremity musculoskeletal discomfort. Participants were assigned to a control group, a breaks-only group, or a breaks-and-exercise group. In the breaks-only group, participants took 5-minute breaks after each 35-minute period of continuous computer use and 7-second microbreaks after each 5-minute period of continuous computer usage. The microbreaks were not administered after 5-minute periods during which participants took "natural" breaks which were of equal or greater duration than the software prompted breaks. The same break and microbreak schedule was applied to the breaks-and-exercise group. Participants in the breaks-and-exercise group performed four 45 -second exercises at the beginning of the 5-minute breaks. The severity of musculoskeletal complaints decreased in the neck and throughout the upper extremity in all groups. The frequency of complaints decreased in the neck and shoulder, and increased in the elbow, wrist, and hands in all groups. After adjustment for sex and age, both intervention groups reported significantly greater recovery from musculoskeletal complaints than the control group. There was no difference in recovery from musculoskeletal discomfort between the intervention groups. Productivity was highest in the breaks-only group.

A randomized controlled clinical trial was performed in van den Heuval's study. Randomized controlled clinical trials with more than 100 participants, including this study by van den Heuval et al. ${ }^{15)}$, have evidence level I research designs. The internal validity of this study was limited to moderate because the measure used for musculoskeletal discomfort has not been proven reliable ${ }^{15,26)}$.

\section{DISCUSSION}

\section{Grades of recommendation}

The strength of evidence of each report was evaluated using the AACPDM method ${ }^{26)}$. The aggregate strength of each intervention was evaluated and grades of recommendation were given based on the CEBM method ${ }^{27,28)}$. Strength of research evidence does not equate to intervention effectiveness.

The aggregate conclusion from the articles appraised is that exercise decreases musculoskeletal discomfort during computer tasks. The overall grade of recommendation for exercise as an intervention to decrease musculoskeletal discomfort during computer tasks is a $\mathrm{C}$, because the evidence does not present consistent level I or II studies and only two level V studies are included (Table 6).

Eight articles confirmed the effectiveness of exercise breaks for reducing musculoskeletal discomfort in computer tasks ${ }^{10-12,14,15,31,38,39) .}$ Only one article, Henning et al., 1997 ${ }^{12)}$, found exercise breaks not beneficial for reducing musculoskeletal discomfort. They found exercise did decrease musculoskeletal discomfort at a small work site, but not at a large worksite. The participants at the large worksite were instructed to give priority to customer inquires over rest/exercise breaks. Therefore, many breaks may have been skipped.

Based on the articles appraised in this review, rest breaks are effective at reducing musculoskeletal discomfort during computer tasks. The overall grade of recommendation for rest breaks as an intervention for decreasing musculoskeletal 
Table 7. Level of evidence summary-Rest breaks

\begin{tabular}{|c|c|c|c|c|c|c|c|c|c|c|c|}
\hline \multirow[b]{2}{*}{ Study } & \multicolumn{7}{|c|}{ Internal validity questions } & \multirow{2}{*}{$\begin{array}{l}\text { Internal } \\
\text { validity } \\
\text { sum }\end{array}$} & \multirow{2}{*}{$\begin{array}{c}\text { Internal } \\
\text { validity } \\
\text { score* }\end{array}$} & \multirow{2}{*}{$\begin{array}{c}\text { Research } \\
\text { design }\end{array}$} & \multirow{2}{*}{$\begin{array}{l}\text { Level of } \\
\text { evidence }\end{array}$} \\
\hline & 1 & 2 & 3 & 4 & 5 & 6 & 7 & & & & \\
\hline \multicolumn{12}{|c|}{ Articles concluding rest breaks are beneficial } \\
\hline Balci, 2003 ${ }^{10)}$ & Yes & Yes & Yes & No & Yes & Yes & Yes & 6 & $\mathrm{~S}$ & II & II-S \\
\hline Ferreira et al., 199734) & Yes & Yes & Yes & No & Yes & No & No & 4 & M & IV & IV-M \\
\hline Galinsky et al., 20009) & Yes & Yes & No & No & Yes & No & Yes & 4 & M & II & II-M \\
\hline Henning et al., 1994 & Yes & Yes & No & No & Yes & No & No & 3 & $\mathrm{~W}$ & IV & IV-W \\
\hline Henning et al., 1997 small worksite ${ }^{12)}$ & Yes & Yes & No & No & Yes & No & No & 3 & $\mathrm{~W}$ & II & II-W \\
\hline McLean et al., 2001 ${ }^{13)}$ & Yes & Yes & Yes & No & Yes & Yes & Yes & 6 & $\mathrm{~S}$ & II & II-S \\
\hline Sundelin \& Hagberg, 19890) & Yes & Yes & Yes & No & No & Yes & Yes & 5 & M & IV & IV-M \\
\hline Swanson \& Sauter, 199241) & Yes & Yes & No & No & No & No & Yes & 3 & $\mathrm{~W}$ & II & II-W \\
\hline van den Heuval et al., 2003 $3^{15)}$ & Yes & Yes & No & No & Yes & Yes & Yes & 5 & M & $\mathrm{I}$ & $\mathrm{I}-\mathrm{M}$ \\
\hline Zwahlen et al., $1984^{38)}$ & Yes & Yes & No & No & No & Yes & No & 3 & $\mathrm{~W}$ & $\mathrm{~V}$ & $\mathrm{~V}-\mathrm{W}$ \\
\hline \multicolumn{12}{|c|}{ Articles concluding rest breaks are not beneficial } \\
\hline Boucsein \& Thum, 199533) & Yes & Yes & No & No & No & No & No & 2 & $\mathrm{~W}$ & IV & IV-W \\
\hline Henning et al., 1997 large worksite & Yes & Yes & No & No & No & No & No & 2 & $\mathrm{~W}$ & IV & IV-W \\
\hline Sundelin et al., 1986 37 ) & Yes & Yes & Yes & No & No & Yes & Yes & 5 & M & IV & IV-M \\
\hline
\end{tabular}

*S=Strong; $\mathrm{M}=$ Moderate; $\mathrm{W}=\mathrm{Weak}^{31)}$.

Grade of Recommendation for Rest Breaks: 27 C.

Grade of Recommendation for No Rest Breaks: 27 D.

discomfort during computer tasks is $\mathrm{C}$ because the evidence does not provide consistent level I or II studies, and includes only one level V study. The quality of evidence has improved since 2000 for rest breaks. All studies published since 2000 on the effect of rest breaks on musculoskeletal discomfort during computer tasks have levels of evidence of I and II and have moderate to strong internal validity (Table 7).

Ten articles confirmed the effectiveness of rest breaks in decreasing musculoskeletal discomfort in computer tasks ${ }^{9-10,12-13,15,33,37-39)}$. Boucsein and Thum ${ }^{32)}$, Sundelin et al. ${ }^{36)}$, and Henning et al. $\left.1997^{12}\right)$ did not find rest breaks effective. Sundelin et al. ${ }^{36)}$ found musculoskeletal discomfort increased during all work periods with no difference in musculoskeletal discomfort between two pause types. Boucsein and Thum ${ }^{32}$ found musculoskeletal discomfort increased over the work day and no significant differences between two work/rest schedules. Henning et al. 19972) found rest breaks did decrease musculoskeletal discomfort at a small work site, but not at a large worksite.

Swanson and Sauter ${ }^{39)}$ found that exercise and rest breaks were equally effective in decreasing stresses of VDT work. Van den Heuval et al. ${ }^{15)}$ found no additional effects of exercises over rest breaks in recovery of musculoskeletal discomfort. Sundelin and Hagberg ${ }^{38)}$ found no significant difference in perceived discomfort between passive breaks, diverting breaks, and exercise breaks. Henning et al. 1997 ${ }^{12}$ ) found exercise breaks were more effective than passive rest breaks in decreasing musculoskeletal discomfort at a small work site but no difference between passive rest breaks and exercise breaks at a large worksite.

\section{Comparison to existing guidelines}

The results of this evidence review on exercise and rest breaks during computer work are consistent with the recommendations of the Occupational Safety and Health Administration (OSHA). OSHA makes the following recommendations: 1) stretch the torso, fingers, hands, and arms frequently throughout the day; 2) take several short rest breaks; 3 ) stand up and walk for a few minutes periodically; 4) alternate computer tasks with non-computer tasks whenever possible; and 5) make small adjustments to the desk chair ${ }^{40)}$.

The Official Disability Guidelines Treatment (ODG) in Workers' Compensation grades the limited evidence for microbreaks as $2 b$ for workers 
with a history of carpal tunnel syndrome. The grade of $2 \mathrm{~b}$ represents randomized controlled trials with moderate quality. ODG recommends 5-minute rest breaks for every 30 minutes of work for computer operators with a history of neck pathology. The ODG does not recommend specific work-rest schedules for any other body regions. The ODG recommends a maximum of fifteen keystrokes per minute for a maximum of two hours per day for computer operators with recent work-related musculoskeletal disorders. Exercise, based on high quality research, is recommended for the treatment of work related musculoskeletal disorders of all etiologies by guidlelines.gov and ODG. ODG and guidlelines.gov suggest stretching may aid in prevention of low back pain ${ }^{41,42)}$. The ODG recommends frequent stretching in cases of carpal tunnel syndrome. However, the ODG and guidelines.gov do not specifically mention brief exercise breaks or workstation exercises during computer work $^{41,42)}$.

\section{Aggregate limitations on internal validity}

Internal validity threats were present in all articles appraised. This was inherent in the dependent variable being measured. Since musculoskeletal discomfort was subjectively reported by participants, the outcome assessor was the subject. Therefore, the outcome assessor could not be blind to intervention status. This lowered the internal validity score of all studies by one point. Internal validity was low in many field studies because the dropout rate was high. This was mostly due to employment attrition, not the studies themselves. The internal validity score was lowered one point in every study in which the dropout rate was $20 \%$ or greater, or if dropout was not reported.

Compliance to the intervention was inconsistent in many of the studies appraised. This may be attributed to employees skipping rest and/or exercise breaks when work demands were high. This weakness is a typical response of employees who have mandatory productivity standards ${ }^{2}$. In the study by Henning et al. 1997, computer operators who worked under an incentive pay system had increased productivity when rest breaks, or rest breaks with exercise, were added to their workday ${ }^{12)}$.

Most of the studies appraised had decreased internal validity because the reliability of the tools used to measure musculoskeletal discomfort was not presented in the articles. The tools may be valid and reliable, but this could not be inferred from the articles. Another reason for low internal validity in the reviewed studies was five $(33 \%)$ of the studies did not include power calculations when statistical significance was not found. In those studies the results should be interpreted with caution since it is unknown if the study had sufficient power to detect a treatment effect. This is a major weakness throughout medical literature. In the review of Moher et al. review of 383 RCT's published in medical journals, they found only $32 \%$ of the trials with negative results reported sample size calculations ${ }^{43)}$. Halpern's review found only $42 \%$ of studies reported power calculations, and only $79 \%$ of trials had adequate power to reliably detect a true treatment effect ${ }^{44)}$.

\section{REFERENCES}

1) Aaras A, Horgen G, Bjorset HH, et al.: Musculoskeletal, visual and psychosocial stress in VDU operators before and after multidisciplinary ergonomic interventions. Appl Ergon, 1998, 29: 335354.

2) Arndt R: Working posture and musculoskeletal problems of video display terminal operators - review and reappraisal. Am Ind Hyg Assoc J, 1983, 44: 437446.

3) Bergqvist U, Wolgast E, Nilsson B, et al.: The influence of VDT work on musculoskeletal disorders. Ergonomics, 1995, 38: 754-762.

4) Demure B, Luippold RS, Bigelow C, et al.: Video display terminal workstation improvement program: I. Baseline associations between musculoskeletal discomfort and ergonomic features of workstations. $\mathbf{J}$ Occup Environ Med, 2000, 42: 783-791.

5) Gerr F, Marcus M, Ensor C, et al.: A prospective study of computer users: I. study design and incidence of musculoskeletal symptoms and disorders. Am J Ind Med, 2002, 41: 221-235.

6) Hunting W, Laubli T, Grandjean E: Postural and visual loads at VDT workplaces. I. Constrained postures. Ergonomics, 1981, 24: 917-931.

7) Sauter SL, Schleifer LM, Knutson SJ: Work posture, workstation design, and musculoskeletal discomfort in a VDT data entry task. Hum Factors, 1991, 33: 151167.

8) Hedge A, Morimoto S, McCrobie D: Effects of keyboard tray geometry on upper body posture and comfort. Ergonomics, 1999, 42: 1333-1349.

9) Galinsky TL, Swanson NG, Sauter SL, et al.: A field study of supplementary rest breaks for data-entry operators. Ergonomics, 2000, 43: 622-638.

10) Balci R, Aghazadeh F: The effect of work-rest 
schedules and type of task on the discomfort and performance of VDT users. Ergonomics, 2003, 46: 455-465.

11) Fenety A, Walker JM: Short-term effects of workstation exercises on musculoskeletal discomfort and postural changes in seated video display unit workers. Phys Ther, 2002, 82: 578-589.

12) Henning R, Jacques $P$, Kissel G, et al.: Frequent short rest breaks from computer work: effects on productivity and well-being at two field sites. Ergonomics, 1997, 40: 78-91.

13) McLean L, Tingley M, Scott R, et al.: Computer terminal work and the benefit of microbreaks. Appl Ergon, 2001, 32: 225-237.

14) Thompson D: Effect of exercise breaks on musculoskeletal strain among data-entry operators: a case study. In: Promoting Health and Productivity in the Computerized Office: Models of Successful Ergonomic Intervention. Sauter S, Dainoff M, Smith M (eds.), London: Taylor and Francis, 1990, pp 118127.

15) van den Heuvel S, de Looze M, Hildebrandt V, et al.: Effects of software programs stimulating regular breaks and exercises on work-related neck and upperlimb disorders. Scand J Work Environ Health, 2003, 29: 106-116.

16) Carter J, Banister E: Musculoskeletal problems in VDT work: a review. Ergonomics, 1994, 37: 16231648 .

17) Arndt R: Working posture and musculoskeletal problems of video display terminal operators - review and reappraisal. Am Ind Hyg Assoc J, 1983, 44: 437446.

18) Tittiranonda P, Burastero S, Rempel D: Risk factors for musculoskeletal disorders among computer users. Occup Med, 1999, 14: 17-38.

19) Korhonen $T$, Ketola R, Toivonen R, et al.: Work related and individual predictors for incident neck pain among office employees working with video display units. Occup Environ Med, 2003, 60: 475-482.

20) Omer S, Ozcan E, Karan A, et al.: Musculoskeletal system disorders in computer users: effectiveness of training and exercise programs. J Back Musculoskeletal Rehabil, 2003-2004, 17: 9-13.

21) Bergqvist $U$, Wolgast $E$, Nilsson $B$, et al.: Musculoskeletal disorders among visual display terminal workers: individual, ergonomic, and work organizational factors. Ergonomics, 1995, 38: 763776.

22) Bernard B, Sauter S, Fine L, et al.: Job task and psychosocial risk factors for work-related musculoskeletal disorders among newspaper employees. Scand J Work Environ Health, 1994, 20: 417-426.

23) Ortiz-Hernandez L, Tamez-Gonzalez S, MartinezAlcantara S, et al.: Computer use increases the risk of musculoskeletal disorders among newspaper office workers. Arch Med Res, 2003, 34: 331-342.
24) Lee K, Swanson N, Sauter S, et al.: A review of physical exercises recommended for VDT operators. Appl Ergon, 1992, 23: 387-408.

25) Lee K, Waikar A: Types of activities and body parts affected in the recommended exercises for VDT operators. J Hum Ergol, 1991, 20: 13-26.

26) Treatment Outcomes Committee. AACPDM methodology to develop systematic reviews of treatment interventions. Revision 1.1, 2004 Version. AACPDM Web site. http://www.aacpdm.org/ resources/systematicReviewsMethodology.pdf. (Accessed Apr. 29, 2005).

27) Levels of evidence and grades of recommendation. Centre for Evidence-based Medicine. http:// www.cebm.net/levels_faq.asp. (Accessed Nov. 17, 2005).

28) Sackett DL, Straus SE, Richardson WS, et al.: Evidence-Based Medicine: How to Practice and Teach EBM. 2nd ed. London: Churchill Livingston, 2000.

29) Butler C, Darrah J: Effects of neurodevelopmental treatment (NDT) for cerebral palsy: an AACPDM evidence report. Dev Med Child Neurol, 2001, 43: 778-790.

30) Polczynski T, Mollinger-Riemann L: Evidence for practice: Literature review on effectiveness of resistance exercise in older adults with knee osteoarthritis. In: American Physical Therapy Association Combined Sections Meeting. New Orleans, LA, 2005.

31) Saltzman A: Computer user perception of the effectiveness of exercise mini-breaks. In: Proceedings of the Silicon Valley Ergonomics Conference and Exposition. Silicon Valley, CA, 1998, pp 147-151.

32) Boucsein W, Thum M: Recovery from strain under different work/rest schedules. In: Proceedings of the Human Factors and Ergonomics Society 39th Annual Meeting. Santa Monica, CA, 1995, 785-788.

33) Ferreira M, de Souza Conceicao G, Nascimento Saldiva P: Work organization is significantly associated with upper extremity musculoskeletal disorders among employees engaged in interactive computer telephone tasks of an international bank subsidiary in Sao Paulo, Brazil. Am J Ind Med, 1997, 31: 468-473.

34) Frequently-asked questions on levels of evidence. Centre for Evidence-based Medicine Website. http:// www.cebm.net/levels_faq.asp. (Accessed November 17, 2005).

35) Henning R, Kissel G, Maynard D: Compensatory rest breaks for VDU operators. Int J Ind Erg, 1994, 14: 234-249.

36) Sundelin G, Hagberg M, Hammarstrom U: The effects of pauses on muscular load and perceived discomfort when working at a VDT word processor. In: Proceedings of the International Scientific Conference: Work with Display Units, Part I. Stolholm, 1986, pp 501-502.

37) Zwahlen H, Hartmann A, Rangarajulu S: Effects of 
rest breaks in continuous VDT work on visual and musculoskeletal comfort/discomfort and performance. In: Human-Computer Interaction. Salvendy G (ed.), Amsterdam: Elsevier, 1984, pp 315-319.

38) Sundelin G, Hagberg M: The effects of different pause types on neck and shoulder EMG activity during VDU work. Ergonomics, 1989, 32: 527-537.

39) Swanson N, Sauter S: The effects of exercise on the health and performance of data entry operators. In: Work with display units '92. Luczak C (ed.), Amsterdam: Elsevier, 1993, pp 288-291.

40) Computer workstations: work process and recognition. U.S. Department of Labor Occupational Safety and
Health Administration Website. http://www.osha.gov/ SLTC/etools/computerworkstations/workprocess.html \#Prolonged. (Accessed Nov. 19, 2005).

41) Official Disability Guidelines Treatment in Workers' Compensation, 3rd ed. Encinitas, CA: Work-Loss Data Institute, 2005.

42) National Guideline Clearinghouse. http://guidelines. gov/. (Accessed Nov. 25, 2005).

43) Moher D, Dulberg C, Wells G: Statistical power, sample size, and their reporting in randomized controlled trials. JAMA, 1994, 272: 122-124.

44) Halpern S: Adding nails to the coffin of underpowered trials. J Rheumatol, 2005, 32: 2065-2066. 